\title{
BACKGROUND ACTIVITY AND VISUAL RESPONSIVENESS OF CAUDATE NUCLEUS NEURONS IN HALOTHANE ANESTHETIZED AND IN AWAKE, BEHAVING CATS
}

\author{
BALÁZS BARKÓCZI, TAMÁS NAGYPÁL, DIÁNA NYUJTÓ, \\ XÉNIA KATONA, GABRIELLA EÖRDEGH, \\ BALÁZS BODOSI, GYÖRGY BENEDEK, \\ GÁBOR BRAUNITZER AND ATTILA NAGY * \\ Department of Physiology, Faculty of Medicine, University of \\ Szeged, Dóm tér 10, Szeged H-6720, Hungary
}

\begin{abstract}
This study focuses on the important question whether brain activity recorded from anesthetized, paralyzed animals is comparable to that recorded from awake, behaving ones. We compared neuronal activity recorded from the caudate nucleus (CN) of two halothaneanesthetized, paralyzed and two awake, behaving cats. In both models, extracellular recordings were made from the $\mathrm{CN}$ during static and dynamic visual stimulation. The anesthesia was maintained during the recordings by a gaseous mixture of air and halothane $(1.0 \%)$. The behaving animals were trained to perform a visual fixation task. Based on their electrophysiological properties, the recorded $\mathrm{CN}$ neurons were separated into three different classes: phasically active (PANs), high firing (HFNs), and tonically active (TANs) neurons. Halothane anesthesia significantly decreased the background activity of the $\mathrm{CN}$ neurons in all three classes. The anesthesia had the most remarkable suppressive effect on PANs, where the background activity was consistently under $1 \mathrm{spike} / \mathrm{s}$. The analysis of these responses was almost impossible due to the extremely low activity. The evoked responses during both static and dynamic visual stimulation were obvious in the behaving cats. On the other hand, only weak visual responses were found in some neurons of halothane anesthetized cats. These results show that halothane gas anesthesia has a marked suppressive effect on the feline $\mathrm{CN}$. We suggest that for the purposes of the visual and related multisensory/sensorimotor electrophysiological exploration of the $\mathrm{CN}$, behaving animal models are preferable over anesthetized ones. (c) 2017 IBRO. Published by Elsevier Ltd. All rights reserved.
\end{abstract}

Key words: caudate nucleus, visual stimulation, halothane anesthetized cats, behaving cats, neuron classification.

\footnotetext{
${ }^{*}$ Corresponding author. Address: Department of Physiology, University of Szeged, Dóm tér 10., POB 427, H-6720 Szeged, Hungary. Fax: + 36-62-545842.

E-mail address: nagy.attila.1@med.u-szeged.hu (A. Nagy).

Abbreviations: CN, caudate nucleus; HFN, high firing neuron; ISI, interspike interval; MAC, minimum alveolar anesthetic concentration; PAN, phasically active neuron; propISI $>2 \mathrm{sec}$, the ratio of the interspike intervals longer than $2 \mathrm{~s}$; PSTH, peristimulus time histogram; SD, standard deviation; TAN, tonically active neuron.
}

\section{INTRODUCTION}

The caudate nucleus $(\mathrm{CN})$, one of the input structures of the basal ganglia, is strongly involved in sensorimotor functions through cortical and subcortical sensorimotor loops in the mammalian brain (McHaffie et al., 2005). Not surprisingly, the $\mathrm{CN}$ neurons are sensitive to both static and dynamic visual stimuli (Rolls et al., 1983; Strecker et al., 1985; Hikosaka et al., 1989; Chudler et al., 1995; Nagy et al., 2003; Nagy et al., 2008; Gombkoto et al., 2011; Rokszin et al., 2011; Vicente et al., 2012; Nagypal et al., 2015). Thus, the $\mathrm{CN}$ seems to belong to those brain structures that have to capacity to sample and evaluate a wide variety of changes in the visual environment.

The domestic cat is a classical mammalian model organism in visual neurophysiology and neuroanatomy. An aspect common to all visual electrophysiological experiments is the need to control for eye movements. This is critical, on the one hand because the spatial position of the visual stimulus has to overlap with the receptive field of the investigated neuron and, on the other hand, visual responses need to be separated from oculomotor activity. The latter is of particular importance here because neurons in the ascending tectofugal visual system, which relay visual information to the $\mathrm{CN}$, also exhibit oculomotor activity (Hikosaka et al., 2000; Munoz and Fecteau, 2002). There are two possible ways to control for the effects of eye movements. The first is to use anesthetized and paralyzed animals, and the second is to train behaving animals to restrict eye movements. Anesthesia, combined with muscle paralysis is a very effective way of stabilizing the eye and thus the location of retinal stimulation. However, this does not allow longterm data collection, and it increases the number of animals that have to be sacrificed. In addition, the anesthetics can influence the neural activity. Although earlier findings suggested that anesthesia and paralysis have only a minor effect on the background activity, visual responsiveness and receptive field structure of cortical neurons (Wurtz, 1969), anesthetics do not necessarily have the same effect in the cortex as in the deeper brain structures (Velly et al., 2007). Furthermore, it has been reported that the basal ganglia are extremely sensitive to anesthesia (Kaisti et al., 2003; Franks and Zecharia, 2011). In an early work in halothane-anesthetized cats, only a small proportion of $\mathrm{CN}$ cells were found to show spontaneous activity (Ben-Ari and Kelly, 1976). In an effort to reduce the number of sacrificed animals, and also to eliminate the potentially confounding effects of anes- 
thetics, electrophysiology has gradually turned toward the use of awake, behaving animals in the last few decades. This tendency has been less marked in visual electrophysiology, because of the long training periods. In such a training period, the animals learn to keep fixation so that the effects of the eye movements can be eliminated, or at least reduced to the minimum (Robinson, 1963; Fuchs and Robinson, 1966; Judge et al., 1980). In our experience, the training and preparation of a single cat is often a challenging process that may take up to 9 months (Nagypal et al., 2014). This naturally raises the question as to whether the costs and benefits are in equilibrium. Assessing the effects of halothane on neural activity in the $\mathrm{CN}$ is important not only for the interpretation and re-evaluation of already existing results, but also for weighing the costs and benefits of utilizing awake, behaving animals in future studies.

\section{EXPERIMENTAL PROCEDURES}

Two awake, behaving and another two halothaneanesthetized, paralyzed, adult domestic cats were used for the experiments, each weighing between 2.5 and $4 \mathrm{~kg}$. Both cats in the chronic (behaving) model were female, and recordings were made between the ages of one and four years. One animal in the anesthetized model was a female and the other was a male, aged two and three years, respectively. Experiments carried out with the anesthetized model lasted for five days.

All experimental procedures were carried out to minimize the number and the discomfort of animals involved, and followed the European Communities Council Directive of 24 November 1986 (86 609 EEC) and the US National Research Council's Guide to the care and Use of Laboratory Animals (National Research Council (U.S.). Committee for the Update of the Guide for the Care and Use of Laboratory Animals et al., 2011). The experimental protocol was approved by the Ethics Committee for Animal Research at the University of Szeged.

\section{Animal preparation and surgery}

All animals were initially anesthetized with ketamine hydrochloride (Calypsol (Gedeon Richter), $30 \mathrm{mg} / \mathrm{kg}$ i. $\mathrm{m}$.). To reduce salivation and bronchial secretion, a subcutaneous injection of $0.2 \mathrm{ml} 0.1 \%$ atropine sulfate was administered preoperatively. All wound edges and pressure points were treated generously with local anesthetic (1\%, procaine hydrochloride). During the whole surgery for both experimental models, and during the recording sessions of anesthetized animals a tracheal tube was used for the delivery of gas anesthetics. The anesthetized animals were artificially ventilated with $1.6 \%$ halothane during surgery, which was reduced to $1.0 \%$ during the recording sessions. In the animals prepared for the awake recordings, the anesthesia was maintained by $1.0 \%$ isoflurane. The depth of the anesthesia was monitored by continuously checking the end-tidal anesthetic concentration and heart rate. The minimum alveolar anesthetic concentration (MAC) values calculated from the endtidal anesthetic readings were kept in the range recommended by Villeneuve and Casanova (Villeneuve and Casanova, 2003). The end-tidal halothane and isoflurane concentration, MAC values and the peak expired $\mathrm{CO}_{2}$ concentrations were monitored with a capnometer (Capnomac Ultima, Datex-Ohmeda, ICN). The peak expired $\mathrm{CO}_{2}$ concentration was kept within the range $3.8-4.2 \%$ by the adjustment of the respiratory rate or volume via the ventilator and trachea-tube system, while the MAC values were set by the adjustment of the gas anesthetic evaporation rate. The body temperature of the animal was maintained at $37^{\circ} \mathrm{C}$ by a computer-controlled, warm water heating blanket. Craniotomy was performed with a dental drill to allow a vertical approach to the target structures.

In the case of the anesthetized animals, both the femoral vein and the trachea were cannulated, and the animals were placed in a stereotaxic headholder. Throughout the surgery the anesthesia was continued with halothane $(1.6 \%)$ in air. The animals were immobilized with an initial 2-ml intravenous bolus of gallamine triethiodide (Flaxedil, $20 \mathrm{mg} / \mathrm{kg}$ ), and artificial ventilation was introduced. The dura mater was preserved, and the skull hole was covered with a $4 \%$ solution of $38^{\circ} \mathrm{C}$ agar dissolved in Ringer's solution. During the recording sessions, a mixture containing gallamine triethiodide $(8 \mathrm{mg} / \mathrm{kg} / \mathrm{h})$, glucose $(10 \mathrm{mg} / \mathrm{kg} / \mathrm{h})$ and dextran $(50 \mathrm{mg} / \mathrm{kg} / \mathrm{h})$ in Ringer lactate solution was infused systemically and continuously at a rate of $4 \mathrm{ml} /$ $\mathrm{h}$. The eye contralateral to the cortical recording site was treated locally with atropine sulfate (1-2 drops, $0.1 \%$ ) and phenylephrine hydrochloride (1-2 drops, $10 \%$ ) to dilate the pupils and block accommodation and to retract the nictitating membranes, respectively. In addition, $a+2$ diopter contact lens was applied. The ipsilateral eye was covered during the recordings with an opaque gauze pad. In order to minimize the effects of anesthesia during the recordings, the halothane concentration was reduced to $1.0 \%$. Recordings were performed between the Horsley-Clarke coordinates anterior 12-16 and lateral $4-6.5 \mathrm{~mm}$, at stereotaxic depths between 13 and $19 \mathrm{~mm}$. The recordings were performed with tungsten microelectrodes (A-M Systems, Inc., USA) with an impedance of 2-4 M 2 .

The surgical preparation of the cats for the behaving experiments started with the insertion of a cannula in the femoral vein and continued with the intubation of the trachea. Then the animals were placed in a stereotaxic headholder. Throughout the surgery, the anesthesia was maintained with $1.6 \%$ halothane in a $2: 1$ mixture of $\mathrm{N}_{2} \mathrm{O}$ and oxygen. Surgical procedures were carried out under aseptic conditions. Before the surgical procedure, a preventive dose of antibiotic was given (1000 mg ceftriaxon, i.m., Rocephin $500 \mathrm{mg}$ (Roche)). A reclosable plastic recording chamber $(20 \mathrm{~mm}$ in diameter) was installed on the skull to protect the microdrive system and extracellular electrodes from damage and pollution. Following this, eight parylene 
isolated platinum-iridium wire-electrodes (diameter: $25 \mu \mathrm{m}$ ) were implanted in the $\mathrm{CN}$ in the first cat and eight formvar insulated Nickel-Chrome wire-electrodes (diameter: $50 \mu \mathrm{m}$ ) in the $\mathrm{CN}$ of the second cat. In the case of the first cat an adjustable microdrive system was applied (a modified Harper-McGinty microdrive, see McKown and Schadt (McKown and Schadt, 2006), and a modified Korshunov microdrive was used for the second animal, see Korshunov, (Korshunov, 1995). The implantation of the electrodes was done according to the HorsleyClarke coordinates (anterior 12-14 mm, lateral 5-6.5 mm at stereotaxic depths between 13-19 mm). In order to monitor the eye movements of the animals, a scleral search coil was implanted into one eye. Although this method was originally developed for primates (Robinson, 1963; Fuchs and Robinson, 1966; Judge et al., 1980), it was later adapted to cats (Pigarev and Rodionova, 1998; Populin and Yin, 1998, 2002; Huxlin and Pasternak, 2004; Tollin et al., 2005; Pigarev and Levichkina, 2011). The scleral coils were made of insulated medical stainless steel hook-up wire (Cooner wire, Owensmouth, CA, USA). The coil was looped 3 times at a diameter of $22 \mathrm{~mm}$.

Additionally, a stainless steel headholder was cemented to the skull for head fixation purposes. On the first five postoperative days, ceftriaxone antibiotic was administered intramuscularly (Rocephine, $50 \mathrm{mg} / \mathrm{kg}$ ). Nalbuphin $(0,25 \mathrm{mg} / \mathrm{kg})$ and $40 \mathrm{ml}$ Ringer's lactate solution were administered until the seventh postoperative day.

\section{Behavioral training of the awake, behaving animals before the recordings}

The detailed description of the implantation and the behavioral training of the animals can be found in our recent methodological paper (Nagypal et al., 2014). Therefore we give only a brief summary here. The duration of the behavioral training before the start of the recordings was a maximum of 9 months, including 3 months of pre- surgery training and 6 months of postsurgery training in each cat. The experimental animals were selected with care, and then they were adapted to the laboratory environment. Animals with intact vision and hearing were selected for the experiments. Outstanding teachability, cooperative behavior and easy adaptation to the laboratory environment were also eligibility criteria. Food deprivation was used on weekdays, and the animals received food only in the laboratory (150$250 \mathrm{~g} /$ day). During the weekends, the animals had access to food in their home cage ad libitum. Water was always available ad libitum in the animals' home cage. The cats were carefully wrapped in a canvas harness, which leaves the head, tail and legs free, and were suspended in the experimental stand. In the following step, the head of the suspended cat was fixed to the stereotaxic frame by the implanted steel headholder. Electromagnetic field was generated by metal coils installed into the wall of the experimental stand in order to record eye movements. The next step of the behavioral training focused on the control of the eye movements. A green fixation point was presented in the center of the CRT monitor, in a square acceptance window of variable size. The size of the fixation point was $0.8 \mathrm{deg}$ in diameter. At the beginning of the training session, the gaze of the animal was directed at the middle of the CRT monitor. During the fixation training, the fixation time was gradually increased from $100 \mathrm{~ms}$ to $1500 \mathrm{~ms}$. The size of the initial fixation window was $10 \times 10 \mathrm{deg}$. During the training period, it was reduced to $5 \times 5 \mathrm{deg}$ in $2.5 \mathrm{deg}$ steps. If the cat held fixation within the acceptance window for a pre-set fixation time, it was rewarded with pulpy food. Sessions (either training or recording) lasted 1 to $2 \mathrm{~h}$ a day, four to five times a week. Neither eye was covered during the recording sessions.

\section{Visual stimulation}

For the experiments conducted with anesthetized animals, stationary random dot patterns and random dot kinematograms were generated with a custom made script in Matlab (MathWorks Inc., Natick, MA), using the Psychophysics Toolbox extension (Brainard, 1997; Pelli, 1997), and presented on an 18-inch computer monitor (refresh rate: $100 \mathrm{~Hz}$ ) placed $57 \mathrm{~cm}$ in front of the animal. The steady-state luminance of the stationary dots was $10 \mathrm{~cd} / \mathrm{m}^{2}$, while the background luminance was $2 \mathrm{~cd} / \mathrm{m}^{2}$. The dynamic stimuli contained 200 white dots, which moved at $5 \mathrm{deg} / \mathrm{s}$. The extension of the stimulation screen was $40.5 \mathrm{deg}$ by $30.5 \mathrm{deg}$. The diameter of each dot was $0.15 \mathrm{deg}$ in diameter. In the stimulation protocol, a single trial consisted of three parts: first, a blank, black screen was presented to obtain background activity without visual stimulation; then a random dot pattern (static stimulus) was presented; finally, the same dots started to move (dynamic stimulus) in random directions at a speed of $5 \mathrm{deg} / \mathrm{s}$. The lengths of these phases were as follows: $1500-m s$ intertrial interval with black screen, 1500 -ms static stimulation, 1500-ms dynamic stimulation. The stimulus sequence was repeated 500 times, thus the duration of a recording period was approximately 40 min recorded from the same electrode position.

Similarly, in the experiments with behaving cats two sorts of visual stimuli were applied: first a static random dot pattern (static), which was followed by a center in or center out flow field (dynamic) stimulus. For visual stimulation and the presentation of the fixation point a standard 18- inch CRT monitor (refresh rate: $100 \mathrm{~Hz}$ ) was placed in front of the animal, at a distance of $57 \mathrm{~cm}$. The size of the stimulation screen was $40.5 \mathrm{deg}$ by $30.5 \mathrm{deg}$. The size of each dot in both the static and the dynamic stimuli was $0.1 \mathrm{deg}$ in diameter. The steady-state luminance of the stationary dots was $10 \mathrm{~cd} /$ $\mathrm{m}^{2}$, while the background luminance was $2 \mathrm{~cd} / \mathrm{m}^{2}$. The speed of the dots in the flow field increased from 0 to $7 \mathrm{deg} / \mathrm{s}$ toward the periphery of the stimulation screen. The stimuli were generated using a custom made script prepared in the Psychophysics Toolbox of Matlab. During the recordings, the effect of the eye movements on the neuronal activity had to be controlled for, so the cats were trained to perform fixation during the stimulation. For this, a fixation point was presented in the center of the CRT monitor. The size of the fixation point was $0.8 \mathrm{deg}$ in diameter. The animal had to look at 
the fixation point and keep its gaze within a square fixation acceptance window during the trials of the visual fixation task. The size of the acceptance window was $5 \times 5 \mathrm{deg}$. Any single trial consisted of four parts. The first part was the intertrial interval itself (black screen) in order to record the background activity. It was followed by a fixation phase, in which only the green fixation dot was presented on the monitor. In the last two phases, beside the fixation point static, and later dynamic visual stimulation was presented. The lengths of the epochs were as follows: 5000-10,000-ms black screen (intertrial interval), 500-1000-ms fixation phase with a green fixation point presented alone on the monitor, 200$1000-m s$ static and $1000-m s$ dynamic visual stimulation. If the cat managed to maintain fixation throughout all the stimulation phases of a single trial, the trial was accepted. To minimize the influence of eye movements on neuronal activity, the trial was aborted immediately if the animal failed to fixate (i.e. the gaze of the animal moved out of the acceptance window). Such trials were not accepted, neither was reward given to the animals in these cases.

\section{Recording and data analysis}

Amplified neuronal activities from the anesthetized cats were recorded at $20-\mathrm{kHz}$ sampling rate and stored for off-line analysis (Datawave SciWorks, Version 5.0, DataWave Technologies Corporation, USA). In the case of behaving cats, eye movements were monitored and recorded with a search coil system (DNI Instruments, Newark, DE, USA). The recording of the eye movements, stimulus presentation, reward delivery, and data collection were coordinated by a custom made software via a 16 channels National Instruments data acquisition card.

The first step of the data analysis was spike sorting. The channels were band-pass filtered $(300$ to $3000 \mathrm{~Hz})$, an amplitude threshold was calculated, and the extracted spikes were clustered automatically based on principal component analysis in NeuroScope, NDManager, KlustaKwik and Klusters, under manual control (Harris et al., 2000; Hazan et al., 2006). The proper discrimination of simultaneously recorded neurons (i.e. spike waveforms) was confirmed by the autocorrelogram of spike-trains and overlaid spike shapes. The dip of the autocorrelograms at 0 timelag indicated that the spike separation was proper. The clusterisation was followed by a neuron-type characterization based on the shape of the autocorrelogram (time resolutions $100 \mathrm{~ms}$ and $1000 \mathrm{~ms}$ ), the level of background discharge rate and propISI $>2 \mathrm{sec}$, defined as the proportion of the summed values over $2 \mathrm{~s}$ divided by the total session time (SchmitzerTorbert and Redish, 2008; Nagypal et al., 2015). Based on these three parameters, the neurons were divided in three groups: phasically active neurons (PANs), high firing neurons (HFNs) and tonically active neurons (TANs), (Nagypal et al., 2015). From the temporal distribution of the action potentials, peristimulus time histograms (PSTHs) were created. The background discharge rate of each neuron was calculated from their activity in the intertrial intervals. Firing rates during static and dynamic stimulation were compared to the background activity with the Wilcoxon matched pairs test. We considered the neuronal activity as a response if it was significantly different $(p<0.05)$ from the mean background activity and if the mean net change was at least 0.1 -fold $(10 \%)$ compared to the background activity. This latter criterion was added to correct for an excessive experimentwise error rate in the statistical testing and try to avoid false positive responses.

To eliminate potential distortions due to the high prevalence of $\mathrm{CN}$ neurons with extremely low background activities, the visual responses of only those $\mathrm{CN}$ neurons were included in the analysis whose background activity was at least 1 spike/s. A question that may arise is why we compared the visual responses to the background activities and not to the fixation activities in behaving cats. One explanation of this is that there was no fixation phase in the stimulation protocol of anesthetized cats at all. The other is the low amount of fixation sensitive units in our neuronal population. Of the $209 \mathrm{CN}$ neurons with background activity higher than $1 \mathrm{~Hz}$, only 53 (25\%) showed significantly different activity from the background during the fixation phase of the paradigm.

Activities were calculated separately for the different stimulation phases. The evoked activities were calculated by averaging all activities in a given stimulation phase. The last $1 \mathrm{~s}$ of the intertrial intervals was considered as background activity, against which the evoked activities were compared. Gross activities show the total mean firing rate during the different stages of stimulation (static or dynamic). The net discharge rates were calculated by subtracting the background activity from the gross activity. The presentation of both gross and net activities is necessary because gross activities provide information about the overall activity of the neurons and the ratio between the gross and net activities demonstrates magnitude of visually evoked responses.

As the Shapiro-Wilk test indicated that the distribution of the studied variables was non-normal, nonparametric statistical tests were used. The background activities recorded from anesthetized and behaving cats were compared at the population level with the MannWhitney rank sum test. All statistical analyses were performed in Matlab (MathWorks Inc., Version 8.3, Natick, MA) and STATISTICA for Windows (StatSoft Inc., Version 12.0, Tulsa, OK) softwares.

\section{RESULTS}

Altogether 206 neurons were recorded from the $\mathrm{CN}$ of the two anesthetized cats ( 83 from the first and 123 from the second cat). Three hundred and forty-four neurons were recorded from the $\mathrm{CN}$ of the two awake, behaving cats (285 from the first and 59 from the second cat). For the purposes of the analysis, the recorded trials were divided into three epochs: static visual stimulation, dynamic visual stimulation and intertrial interval without any visual stimulation. Background activity was calculated from the intertrial intervals and the stimulated 
Table 1. Descriptive statistics of background and visually evoked activities of $\mathrm{CN}$ neurons from awake, behaving and anesthetized animals

\begin{tabular}{|c|c|c|c|c|c|c|c|c|c|c|c|c|c|}
\hline & \multicolumn{6}{|c|}{ Awake } & \multicolumn{6}{|c|}{ Anesthetized } & \\
\hline & $\mathrm{N}$ & Mean & Median & SD & Min & Max & $\mathrm{N}$ & Mean & Median & SD & Min & Max & \\
\hline All & 344 & 5.19 & 2.13 & 6.87 & 0.01 & 37.91 & 206 & 1.58 & 0.45 & 2.29 & 0.01 & 13.86 & \multirow[t]{8}{*}{ Background } \\
\hline$\geq 1 \mathrm{~Hz}$ & 209 & 8.32 & 6.21 & 7.27 & 1.02 & 37.91 & 81 & 3.60 & 2.75 & 2.56 & 1.04 & 13.86 & \\
\hline HFNs & 88 & 14.60 & 12.87 & 6.96 & 4.67 & 37.91 & 85 & 2.56 & 1.70 & 2.53 & 0.10 & 13.86 & \\
\hline PANs & 219 & 1.39 & 0.60 & 1.89 & 0.01 & 15.42 & 73 & 0.14 & 0.11 & 0.12 & 0.01 & 0.78 & \\
\hline TANs & 28 & 5.35 & 5.82 & 2.53 & 1.26 & 10.61 & 43 & 2.21 & 1.37 & 2.57 & 0.13 & 13.14 & \\
\hline$\geq 1 \mathrm{~Hz} \mathrm{HFNs}$ & 88 & 14.60 & 12.87 & 6.96 & 4.67 & 37.91 & 56 & 3.64 & 3.10 & 2.50 & 1.04 & 13.86 & \\
\hline$\geq 1 \mathrm{~Hz}$ PANs & 85 & 3.03 & 2.44 & 2.17 & 1.02 & 15.42 & 0 & - & - & - & - & - & \\
\hline$\geq 1 \mathrm{~Hz}$ TANs & 28 & 5.35 & 5.82 & 2.53 & 1.26 & 10.61 & 25 & 3.49 & 2.63 & 2.72 & 1.04 & 13.14 & \\
\hline$\geq 1 \mathrm{~Hz} \geq 10 \%$ Increase Gross & \multirow[t]{2}{*}{51} & 12.86 & 8.15 & 12.17 & 1.46 & 43.86 & \multirow[t]{2}{*}{3} & 3.23 & 2.03 & 2.88 & 1.15 & 6.51 & \multirow[t]{6}{*}{ Static } \\
\hline$\geq 1 \mathrm{~Hz} \geq 10 \%$ Increase Net & & 4.33 & 2.60 & 4.73 & 0.34 & 19.52 & & 0.47 & 0.46 & 0.37 & 0.11 & 0.85 & \\
\hline$\geq 1 \mathrm{~Hz} \geq 10 \%$ Decrease Gross & \multirow[t]{2}{*}{32} & 4.92 & 3.69 & 4.96 & 0.52 & 21.52 & \multirow[t]{2}{*}{2} & 4.85 & 4.85 & 1.91 & 3.50 & 6.20 & \\
\hline$\geq 1 \mathrm{~Hz} \geq 10 \%$ Decrease Net & & -2.37 & -1.09 & 3.24 & -15.91 & -0.20 & & -1.85 & -1.85 & 0.37 & -2.11 & -1.59 & \\
\hline$\geq 1 \mathrm{~Hz}$ No change Gross & \multirow[t]{2}{*}{126} & 10.22 & 8.11 & 7.89 & 1.00 & 41.37 & \multirow[t]{2}{*}{76} & 3.58 & 2.77 & 2.60 & 1.05 & 14.23 & \\
\hline$\geq 1 \mathrm{~Hz}$ No change Net & & 1.73 & 0.99 & 2.86 & -3.20 & 13.38 & & 0.03 & 0.01 & 0.16 & -0.32 & 1.09 & \\
\hline$\geq 1 \mathrm{~Hz} \geq 10 \%$ Increase Gross & \multirow[t]{2}{*}{22} & 14.11 & 12.10 & 10.98 & 1.52 & 33.32 & \multirow[t]{2}{*}{0} & - & - & - & - & - & \multirow[t]{6}{*}{ Dynamic } \\
\hline$\geq 1 \mathrm{~Hz} \geq 10 \%$ Increase Net & & 3.82 & 2.53 & 3.66 & 0.48 & 13.53 & & - & - & - & - & - & \\
\hline$\geq 1 \mathrm{~Hz} \geq 10 \%$ Decrease Gross & \multirow[t]{2}{*}{3} & 5.86 & 4.85 & 4.86 & 1.58 & 11.15 & \multirow[t]{2}{*}{10} & 2.78 & 2.06 & 1.88 & 1.06 & 6.93 & \\
\hline$\geq 1 \mathrm{~Hz} \geq 10 \%$ Decrease Net & & -0.89 & -0.96 & 0.40 & -1.26 & -0.45 & & -0.68 & -0.44 & 0.50 & -1.62 & -0.26 & \\
\hline$\geq 1 \mathrm{~Hz}$ No change Gross & \multirow[t]{2}{*}{184} & 8.66 & 6.13 & 7.86 & 0.70 & 44.61 & \multirow[t]{2}{*}{71} & 3.63 & 2.90 & 2.58 & 1.03 & 13.20 & \\
\hline$\geq 1 \mathrm{~Hz}$ No change Net & & 0.55 & 0.27 & 1.46 & -2.65 & 8.05 & & 0.02 & 0.03 & 0.17 & -0.73 & 0.46 & \\
\hline
\end{tabular}

Not only the background activities, but the evoked responses and the number of visually responsive CN neurons were smaller in case of anesthetized animals. PAN - phasically active neuron, HFN - high firing neuron, TAN - tonically active neuron. The most noteworthy finding is that no PANs with a background activity higher than $1 \mathrm{~Hz}$ were detected in the group of anesthetized animals. There was also no such neuron in the anesthetized recordings, evoked response of which increased more than $10 \%$ during dynamic visual stimulation. All: all neurons; $>1 \mathrm{~Hz}$ : neurons with a background activity equal to or higher than 1 spike/s; $\geq 10 \%$ : evoked responses with a change equal to or higher than 10 percent of the background activity; Increase, Decrease, No change: indicates how the evoked responses changed upon stimulation; Gross, Net: gross and net activities. Gross activity is the averaged firing frequency during the entire stimulation session, while net activity is calculated by subtracting the evoked gross activity from the background firing frequency; Background, Static, Dynamic: trial phases; N: sample size. Discharge rates are in spikes/s. 
A
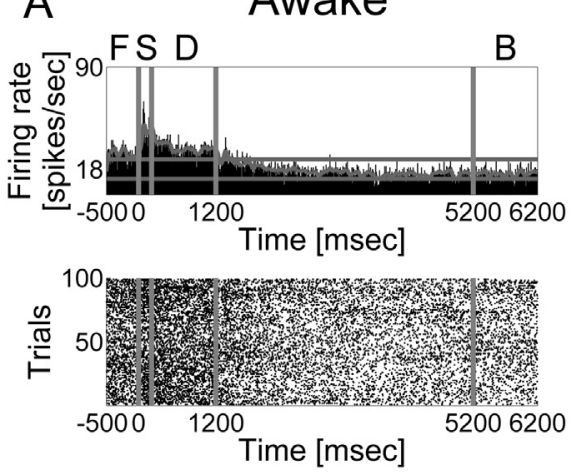

Fig. 1. Peristimulus time histograms and raster plots from awake (A) and anesthetized (B) models. Each panel of the figure contains a PSTH (top) and a raster plot (bottom) to represent the activity of a neuron. Vertical gray lines denote the boundaries between the different phases of the stimulation protocol. In the case of behaving cats the paradigm contained the following phases: fixation phase $(\mathrm{F} ;-500$ to $0 \mathrm{~ms}$ ), static visual stimulation (S; 0 to $200 \mathrm{~ms}$ ), dynamic visual stimulation (D; 200 to $1200 \mathrm{~ms}$ ), reward phase and the following intertrial interval (from $1200 \mathrm{~ms}$ to $6200 \mathrm{~ms}$ ). Background firing rate was calculated from the last second of intertrial interval (B; 5200 to $6200 \mathrm{~ms}$ ). In the case of anesthetized cats the stimulation protocol contained the following phases: static visual stimulation (S; 0 to $1500 \mathrm{~ms}$ ), dynamic visual stimulation (D; 1500 to $3000 \mathrm{~ms}$ ), intertrial interval (3000 to $4500 \mathrm{~ms}$ ). The last second of PSTH corresponds to the background discharge rate (B; 3500 to $4500 \mathrm{~ms}$ ). Trial sessions are also highlighted with capital letters on the top of the PSTH graphs. The abscissa denotes the time in milliseconds. The ordinate denotes the number of trials (bottom) and the activity of the neuron in spikes per seconds (top) The lower value on the ordinate of PSTHs indicates the average background discharge rate during the whole recording, while the continuous gray curve is a smoothed curve of the activity, and the horizontal gray lines indicate $\pm 2 \mathrm{SD}$ of the average background discharge rate. Note that the scale of the ordinate on Part A is much wider than on Part B thus the activities recorded from behaving cat are much higher than those recorded from the anesthetized animals.

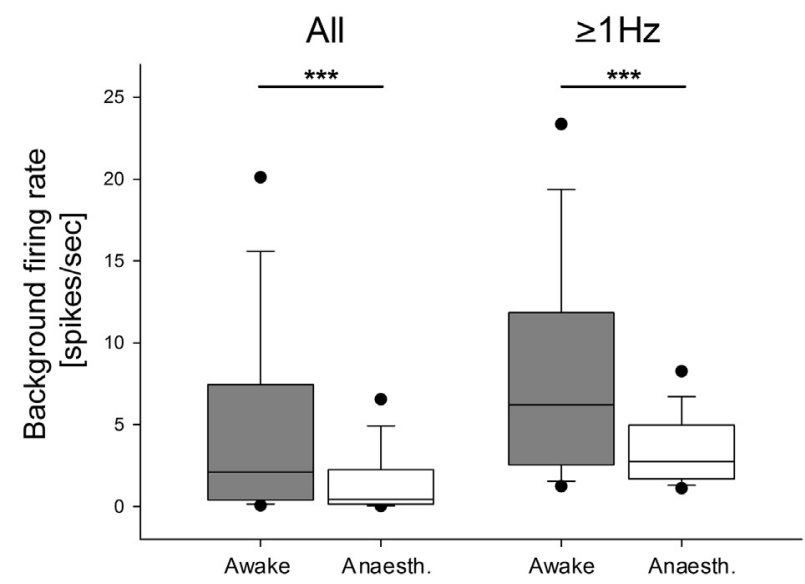

Fig. 2. Neuronal activity in the $\mathrm{CN}$ of awake and anesthetized cats. The box plots on the left side show the background discharge rates of all of the analyzed $\mathrm{CN}$ neurons (All). The two box plots on the right show the background activities of neurons with a discharge rate higher than 1 spike/s $(\geq 1 \mathrm{~Hz}$ ). The gray boxes show the results from behaving cats while the white boxes contains the results from anesthetized cats. The lower margin of the boxes indicates the 25th percentile, the line within the boxes marks the median, and the upper margin of the box indicates the 75th percentile. Whiskers (error bars) above and below the box indicate the 90th and 10 th percentiles. The outliers (dots) represent the 95th and 5th percentiles. All of the presented differences are statistically significant $(p<0.001)$.

activities were compared to this activity. The summary of the detailed descriptive statistics of background and visually evoked activities of $\mathrm{CN}$ neurons from awake, behaving and anesthetized animals can be seen in Table 1.

Fig. 1 shows the peristimulus time histograms and raster plots of two $\mathrm{CN}$ neurons. The first neuron was recorded from an awake (Part A) and the second (Part B) from an anesthetized cat. Not only the background activity, but the evoked gross activities are higher in case of a neuron recorded from behaving animal.

\section{Background activities}

The background activity of the $\mathrm{CN}$ neurons from awake, behaving animals is significantly higher (Mann-Whitney $U, p<0.001$ ) than that of the $\mathrm{CN}$ neurons recorded from anesthetized cats (Fig. 2 All; see descriptive statistical data in Table 1). To eliminate potential distortions due to the high prevalence of $\mathrm{CN}$ neurons with extremely low background activity, a separate analysis was performed after the exclusion of neurons with a background activity under 1 spike/s. In this way, 135 neurons from the awake $(39.2 \%)$ and 125 neurons $(60.7 \%)$ from the anesthetized model were excluded. The remarkably higher prevalence of background activities under $1 \mathrm{~Hz}$ in the anesthetized model were obvious already at this point. The comparison of the background activities higher than $1 \mathrm{~Hz}$ (Fig. $2>1 \mathrm{~Hz}$ neurons) revealed significantly higher activity of $\mathrm{CN}$ neurons recorded from the behaving model (Mann-Whitney $U, p<0.001$; see descriptive statistical data in Table 1).

\section{Responses to static visual stimulation}

The proportion of neurons responsive to static stimulation was much higher in the behaving cats. The gross and net activity increments during static stimulation were notably stronger in the awake, behaving animals than in the anesthetized ones (see descriptive statistical data in Table 1).

The magnitude of the net decrements in activity during static visual stimulation were also more obvious in the behaving model (see descriptive statistical data in Table 1).

\section{Responses to dynamic visual stimulation}

Similarly to the responsiveness to static stimulation, the proportion of neurons responsive to dynamic stimulation was higher in the behaving cats. Activity increments were evident in the behaving animals (see descriptive statistical data in Table 1), however, no such responses were observed in the anesthetized animals at all. The 


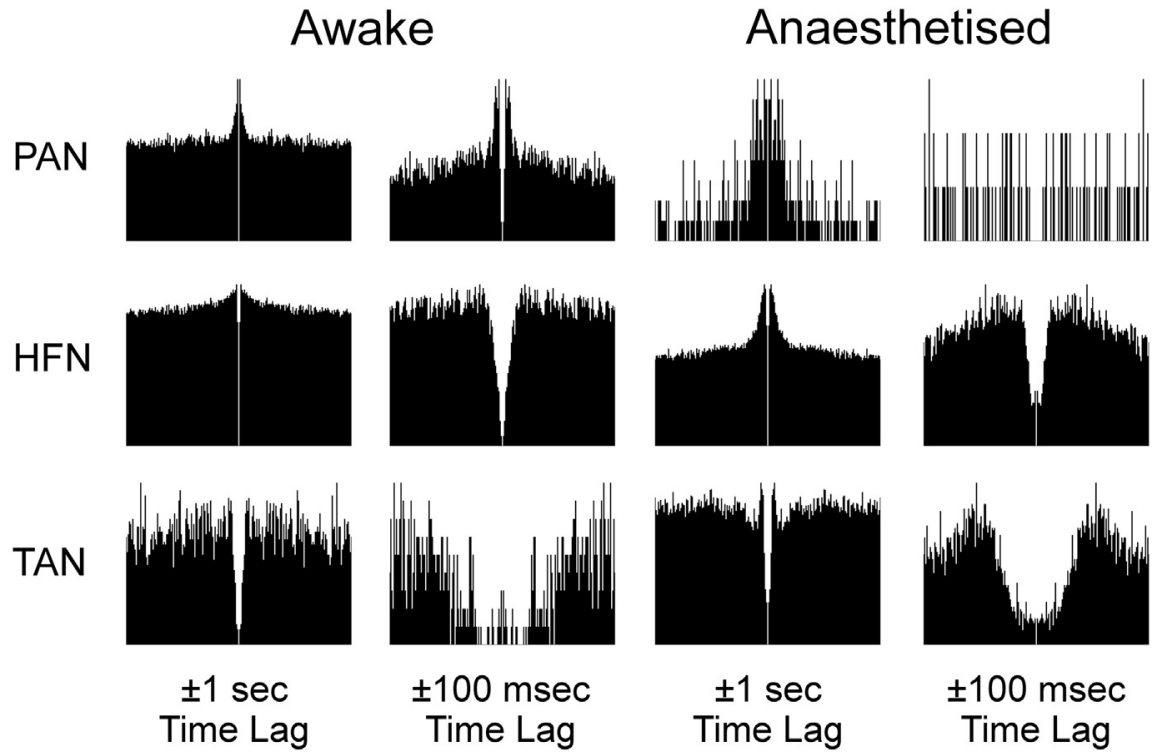

Fig. 3. Representative autocorrelograms of the $\mathrm{CN}$ neurons. $\mathrm{CN}$ neurons were classified on the basis of the shape of their autocorrelograms (at $100 \mathrm{~ms}$ and $1 \mathrm{~s}$ time resolutions), propISI $>2 \mathrm{sec}$ and the background discharge rate in three big groups as follows: PAN - phasically active neuron, HFN - high firing neuron, TAN - tonically active neuron. Neurons belonging to each group have characteristic autocorrelogram. PANs are usually characterized by peaky autocorrelogram and low background discharge rates, HFNs have autocorrelograms with a blunt peak and TANs are characterized by a deep gap in the autocorrelogram. The six representative autocorrelograms on the left side are from awake animals, while the six illustrative panels on the right side are from anesthetized ones, each of them at two different time resolutions.

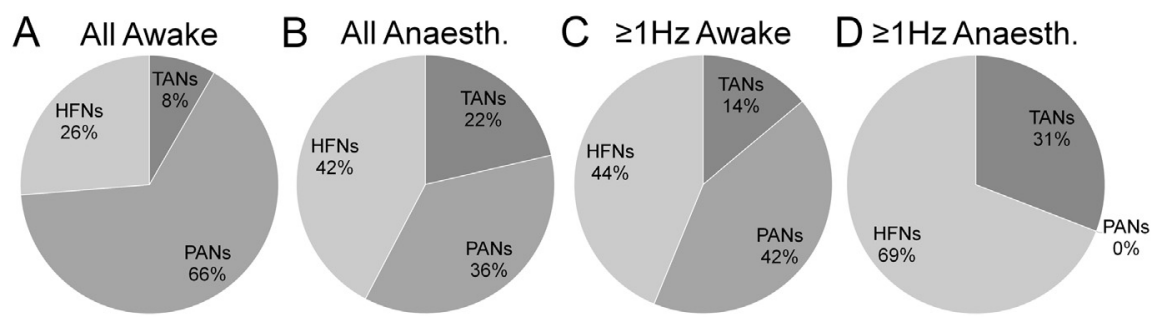

Fig. 4. Pie charts of the distribution of neuron types. Pie charts demonstrate the distribution of neuron types (PAN, HFN and TAN) in the CN in awake (A) and in anesthetized cats (B). The PAN, HFN, TAN labels indicate the different neuronal types, as follows PAN - phasically active neuron, HFN - high firing neuron, TAN - tonically active neuron. Panels $C$ and $D$ characterize the same distribution of $\mathrm{CN}$ neurons with a background discharge rate over $1 \mathrm{~Hz}$. Note that PANs with a background activity over 1 spike/s disappeared during halothane anesthesia.

magnitude of the net decrements in activity during dynamic visual stimulation did not seem to differ by paradigm. It must be mentioned, though, that the sample sizes were quite low (see descriptive statistical data in Table 1).

\section{Electrophysiological groups in the $\mathrm{CN}$ - Phasically active neurons are silent in anesthesia}

Based on their discharge properties (see Recording and Data Analysis) we could classify the recorded $\mathrm{CN}$ neurons in three groups (Fig. 3): phasically active neurons (PANs), high firing neurons (HFNs), tonically active neurons (TANs).

In the $\mathrm{CN}$ of the awake, behaving animals the vast majority of the cells were PANs $(N=219,63.7 \%)$.
Much smaller numbers of HFNs $(N=88,25.6 \%)$ and TANs $(N=28$, $8.1 \%)$ were found. Surprisingly, in the anesthetized animals the HFN group was the most numerous $(N=85, \quad 41.3 \%)$. Fewer PANs ( $N=73,35.4 \%$ ) and even fewer TANs $(N=43,20.9 \%)$ were found (Fig. 4 A, B). A few of the recorded $C N$ neurons could not be categorized this way. This uncategorized group contained 9 units $(2.6 \%)$ from the awake and 5 units $(2.4 \%)$ from anesthetized animals.

Fig. 5 shows the background activities of the different neuron groups in behaving and anesthetized cats. Note that these activities were much lower in all groups recorded from anesthetized cats (see descriptive statistical data in Table 1). The statistical analysis revealed that background activities of all groups were significantly higher in the awake, behaving animals (Mann-Whitney $U, p<0.001$ ).

Of the 135 neurons excluded from the analysis in the awake model because of their low background activity, only one was an uncategorized unit, and the others were all PANs $(N=134)$. On the other hand, the 125 units, which were excluded in the anesthetized model, showed a more varied distribution: $\quad$ PAN $(N=73)$; uncategorized $\quad(N=5) ; \quad$ HFN $(N=29) ; \quad$ TAN $\quad(N=18)$. The prevalence of the neurons belonging to the different electrophysiological groups after the exclusion of the above units can be seen in Fig. $4 C, D$. It is clear that in the anesthetized model, all PANs were excluded by applying the $1 \mathrm{~Hz}$ cut-off criterion. The statistical analysis revealed that the background activities of the HFN and TAN groups were significantly higher in the awake, behaving animals (Mann-Whitney $U, p<0.005$ ).

\section{DISCUSSION}

To our knowledge this is the first study, which compares the neuronal activities recorded from the $\mathrm{CN}$ in two different animal models in anesthetized, paralyzed and in awake, behaving cats. Our results clearly demonstrated that halothane anesthesia could considerably suppress the background activity and visual responses in the $\mathrm{CN}$ of the feline brain. 


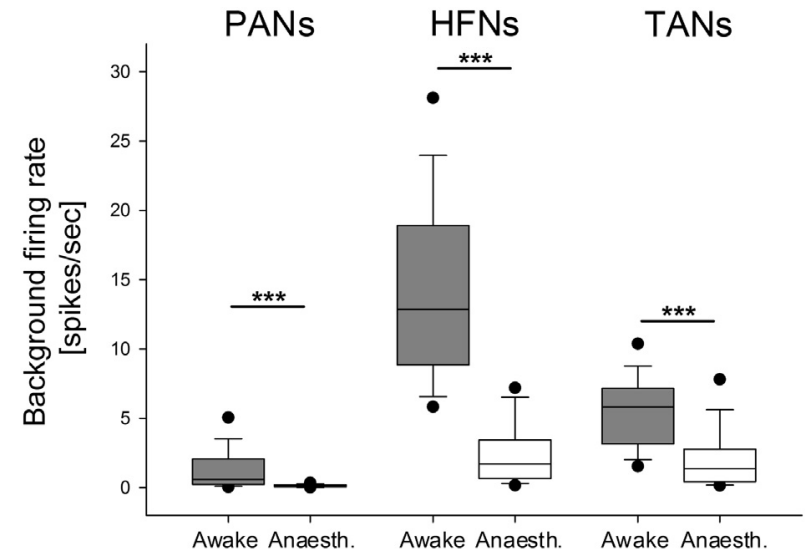

Fig. 5. Background activities of all PANs, HFNs and TANs in behaving and anesthetized cats. The background discharge rates of PANs, HFNs and TANs. The conventions are the same as in Fig. 2. Note that the background activities of each electrophysiological group were significantly higher in the behaving model $(p<0.001)$.

In the case of background discharge rates, the majority $(60.7 \%)$ of the recorded $\mathrm{CN}$ units showed less than $1 \mathrm{~Hz}$ activity in the anesthetized model. This percentage was much lower in the behaving cats $(39.2 \%)$. Because of the low activities, we had to exclude these units from the further analysis. Moreover, the activity changes upon the different kinds of visual stimulation were in most cases very weak in the anesthetized animals. The maximal net response was $2.1 \mathrm{~Hz}$ in the anesthetized animals, while it was much higher in the awake, behaving model (maximal net response $19.5 \mathrm{~Hz}$ ). These results throw light on why it is so difficult to find any kind of activity in the $\mathrm{CN}$ of the anesthetized cats, not to mention proper visual responses. It is also important to see that earlier studies used simple dynamic geometric forms (spots, dots, bars) and drifting sinewave gratings (Pouderoux and Freton, 1979; Strecker et al., 1985; Nagy et al., 2003; Nagy et al., 2006; Nagy et al., 2008; Gombkoto et al., 2011), which are even more adequate visual stimuli for the $\mathrm{CN}$ neurons than our random dot patterns and kinematograms in the present study. According to the literature, a noteworthy proportion of the $\mathrm{CN}$ neurons show direction preference and spatio-temporal selectivity, however, multielectrode recordings allow the recording of several neurons simultaneously, including neurons with preference for different directions and spatio-temporal properties. The most remarkable advantage of the stimuli applied in the present study is that the effects of spatiotemporal and direction preference are minimized. Beyond the lack of halothane anesthesia and immobilization, our behaving model differed from anesthetized models also in a third way: both eyes of the animal were open during stimulation. In anesthetized models during visual stimulation the contralateral eye was opened, while the other eye was covered. Monocular stimulation is essential in anesthetized/paralyzed animal models in order to prevent binocular inhibition that results from ocular misalignment during paralysis. This monocular-binocular difference could influence the magnitude of visual responses, but such a strong difference as seen in this study is not likely to stem from this factor only. Furthermore, the difference in the background discharge rates is against this explanation. Muscle tone, arousal and goal-oriented movements can also increase the activity of the $\mathrm{CN}$ but these cannot be only the explanation for the stronger responsiveness of the $\mathrm{CN}$ neurons in the behaving model.

Fixation can also influence the neuronal activities in the central nervous system. Fixation can be considered to reflect at least some level of attention, and attention is known to influence the activity of the visually responsive parts of the mammalian brain (Rajkai et al., 2008). If we assume that anesthetics can reduce or eliminate attention, this lack of attention may be the reason for the reduction of visual responsiveness in the anesthetized cats. However, it is not likely to lead to such a strong difference as seen in this study. As stated earlier, the large majority of the analyzed CN units were not active during fixation - only one quarter of them showed significant activity changes in the fixation phase of the paradigm. It is also known that the superior colliculi, which comprise the main subcortical source of visual input to the basal ganglia, can also be suppressed during fixation (Sumner et al., 2006). The superior colliculi project to the lateral posterior-pulvinar and the lateral medial-suprageniculate nuclei complex of the thalamus, which are assumed to be the main subcortical visual inputs to the $\mathrm{CN}$. While these thalamic inputs are predominantly excitatory (Hoshino et al., 1997; Hoshino et al., 2000; Hoshino et al., 2009) the lack of excitatory inputs during fixation cannot explain the increased activity in the $\mathrm{CN}$ in behaving animals.

In the intertrial intervals, where background discharge rates were measured, there was no fixation task, that is, the animals were allowed to perform eye movements. In these cases, because of the lack of fixation, the effects of the eye movements on the neuronal activities could also be observed. In our previous study we found no or only very weak correlation between the neuronal activities and the normalized amplitude of the eye movements in the $\mathrm{CN}$. This suggests that the activity of the $\mathrm{CN}$ neurons in the intertrial intervals is not affected significantly by activity changes due to eye movements (Nagypal et al., 2015).

It is noteworthy to mention that the anesthetized cats were cycloplegic and the awake ones had normal pupils and lenses but in the case of anesthetized cats the visus was corrected with appropriate lenses. As the background luminance was the same for the two models and the same CRT monitor was used, these do not explain the observed difference either.

In our previous study, we managed to categorize neurons from the feline $\mathrm{CN}$ based on their electrophysiological properties in behaving cats (Nagypal et al., 2015). This categorization was adapted in the present study to the $\mathrm{CN}$ neurons recorded from anesthetized cats. Although the neuronal activities and the number of spikes are quite different in the two models, the shapes of the autocorrelograms of PANs, TANs and HFNs are basically the same, which suggests that the temporal spiking characteristics of the $\mathrm{CN}$ neurons are 
very similar in the two models. Similarly to earlier findings (Schmitzer-Torbert and Redish, 2004; Schmitzer-Torbert and Redish, 2008; Kubota et al., 2009; Gage et al., 2010; Thorn et al., 2010; Barnes et al., 2011; Stalnaker et al., 2012), PANs, TANs and HFNs were found in both models. The ratio of uncategorizable neurons (under $3 \%$ was also similar. Earlier studies suggested a strong correspondence between the three biggest anatomical (medium spiny, cholinergic and parvalbumin immunpositive GABAergic interneurons) and electrophysiological (PAN, TAN, HFN) groups of the CN neurons. Most probably the PAN neurons correspond to the medium spiny projection neurons, HFNs to the parvalbumin immunpositive GABAergic interneurons and the TANs to the cholinergic interneurons (Kawaguchi, 1993; Wilson, 1993; Kawaguchi et al., 1995; Mallet et al., 2005; Tepper et al., 2010). In line with the results of earlier studies in other species, the majority of the recorded $\mathrm{CN}$ units in the behaving model were PANs in our sample $(64 \%)$. In other species, $77-97 \%$ of the striatal projection neurons belong to this presumably GABAergic cluster (Kemp and Powell, 1971; Luk and Sadikot, 2001). Similarly to earlier results, HFNs $(25 \%)$ and TANs $(8 \%)$ were much less prevalent in our sample (Phelps et al., 1985; Luk and Sadikot, 2001). It must be noted that PANs are often difficult to detect because of their extremely low background activity (Lau and Glimcher, 2007), which means that the sixty-four percent finding may be an underestimation due to undersampling, which, in turn, can also have an effect on the estimations of the two other groups. In contrast to these, the highest amount $(41 \%)$ of the recorded $\mathrm{CN}$ neurons from anesthetized feline model was classified as HFN. Fewer neurons were in the PAN (35\%) group and even fewer in the TAN (21\%) group.

The difference between the anesthetized and behaving feline model is striking. Halothane seems to reduce the background activity of PANs so strongly that it makes a valid analysis impossible. Knowing that, it is clear why these cells are so difficult to detect and record in anesthetized models. We argue that the most important finding of the present study is that the PANs, which seem to correspond to the medium spiny projection neurons (the most prevalent neuron type in the $\mathrm{CN}$ ), are almost silent under anesthesia, and thus these neurons cannot be investigated in anesthetized, paralyzed cats without any afferent stimulation. Furthermore, the activity of the HFNs and TANs was also strongly suppressed in the anesthetized cats, in contrast to the behaving model. In the light of these findings, it has also become clear that the neurons we reported in an earlier study of ours (Nagy et al., 2003) could not be of the medium spiny type - a question we could not agree on at that time.

To summarize our findings, we demonstrated marked and significant differences between the neuronal activities recorded from the feline $\mathrm{CN}$ in an anesthetized and a behaving model. The halothane gas anesthesia and the immobilization significantly suppressed the neuronal activity and the number of visually responsive neurons in the $\mathrm{CN}$. Our results also show that the analysis of PANs (most probably these are the medium spiny projection $\mathrm{CN}$ neurons, the dominant neuronal population in the $\mathrm{CN}$ ) is almost impossible in the anesthetized model because of their extremely low activity. It is clear that to work with behaving cats in visual electrophysiology is much more difficult than to work with anesthetized cats, mostly because of the lengthy preparatory period. However, based on the presented results we suggest that the benefits outweigh the costs, and if PANs are studied, a behaving model can almost be considered a sine qua non of success.

\section{AUTHOR CONTRIBUTIONS}

AN, GYB and GE designed the study; BBa, TN, DN, GE and $\mathrm{XK}$ performed the assessment and documented the findings; $\mathrm{BBa}, \mathrm{BBo}, \mathrm{TN}, \mathrm{DN}$ and $\mathrm{XK}$ analyzed the data; $\mathrm{AN}, \mathrm{GB}$ and $\mathrm{BBa}$ organized the study and wrote the manuscript.

Acknowledgements-The authors thank to Siposné Gabriella Dósai-Molnár, Rozalinda Pose and László Rácz for the technical assistance and to Robert Averkin for the microdrives and the wire electrodes. The authors declare no competing financial interests. This work was supported by Hungarian Brain Research Program Grant KTIA_13_NAP-A-I/15. XK was supported by the ÚNKP-162 New National Excellence Program of the Ministry of Human Capacities, Hungary.

\section{REFERENCES}

Barnes TD, Mao JB, Hu D, Kubota Y, Dreyer AA, Stamoulis C, Brown EN, Graybiel AM (2011) Advance cueing produces enhanced action-boundary patterns of spike activity in the sensorimotor striatum. J Neurophysiol 105:1861-1878.

Ben-Ari Y, Kelly JS (1976) Dopamine evoked inhibition of single cells of the feline putamen and basolateral amygdala. J Physiol 256:1-21.

Brainard DH (1997) The Psychophysics Toolbox. Spat Vis 10:433-436.

Chudler EH, Sugiyama K, Dong WK (1995) Multisensory convergence and integration in the neostriatum and globus pallidus of the rat. Brain Res 674:33-45.

Franks NP, Zecharia AY (2011) Sleep and general anesthesia. Can J Anaesth 58:139-148.

Fuchs AF, Robinson DA (1966) A method for measuring horizontal and vertical eye movement chronically in the monkey. J Appl Physiol 21:1068-1070.

Gage GJ, Stoetzner CR, Wiltschko AB, Berke JD (2010) Selective activation of striatal fast-spiking interneurons during choice execution. Neuron 67:466-479.

Gombkoto P, Rokszin A, Berenyi A, Braunitzer G, Utassy G, Benedek G, Nagy A (2011) Neuronal code of spatial visual information in the caudate nucleus. Neuroscience 182:225-231.

Harris KD, Henze DA, Csicsvari J, Hirase H, Buzsaki G (2000) Accuracy of tetrode spike separation as determined by simultaneous intracellular and extracellular measurements. J Neurophysiol 84:401-414.

Hazan L, Zugaro M, Buzsaki G (2006) Klusters, NeuroScope, NDManager: a free software suite for neurophysiological data processing and visualization. J Neurosci Methods 155:207-216.

Hikosaka O, Sakamoto M, Usui S (1989) Functional properties of monkey caudate neurons. II. Visual and auditory responses. J Neurophysiol 61:799-813.

Hikosaka O, Takikawa Y, Kawagoe R (2000) Role of the basal ganglia in the control of purposive saccadic eye movements. Physiol Rev 80:953-978. 
Hoshino K, Eordegh G, Nagy A, Benedek G, Norita M (2009) Overlap of nigrothalamic terminals and thalamostriatal neurons in the feline lateralis medialis-suprageniculate nucleus. Acta Physiol Hung 96:203-211.

Hoshino K, Hicks TP, Hirano S, Norita M (2000) Ultrastructural organization of transmitters in the cat lateralis medialissuprageniculate nucleus of the thalamus: an immunohistochemical study. J Comp Neurol 419:257-270.

Hoshino K, Hicks TP, Meguro R, Hirano S, Kase M, Norita M (1997) Cholinergic innervation of the lateralis medialis-suprageniculate nuclear complex (LM-Sg) of the cat's thalamus: a double labeling immunohistochemical study. Brain Res 747:151-155.

Huxlin KR, Pasternak T (2004) Training-induced recovery of visual motion perception after extrastriate cortical damage in the adult cat. Cereb Cortex 14:81-90.

Judge SJ, Richmond BJ, Chu FC (1980) Implantation of magnetic search coils for measurement of eye position: an improved method. Vision Res 20:535-538.

Kaisti KK, Langsjo JW, Aalto S, Oikonen V, Sipila H, Teras M, Hinkka S, Metsahonkala L, Scheinin H (2003) Effects of sevoflurane, propofol, and adjunct nitrous oxide on regional cerebral blood flow, oxygen consumption, and blood volume in humans. Anesthesiology 99:603-613.

Kawaguchi Y (1993) Physiological, morphological, and histochemical characterization of three classes of interneurons in rat neostriatum. J Neurosci 13:4908-4923.

Kawaguchi Y, Wilson CJ, Augood SJ, Emson PC (1995) Striatal interneurones: chemical, physiological and morphological characterization. Trends Neurosci 18:527-535.

Kemp JM, Powell TP (1971) The structure of the caudate nucleus of the cat: light and electron microscopy. Philos Trans R Soc Lond B Biol Sci 262:383-401.

Korshunov VA (1995) Miniature microdrive for extracellular recording of neuronal activity in freely moving animals. J Neurosci Methods $57: 77-80$.

Kubota Y, Liu J, Hu D, DeCoteau WE, Eden UT, Smith AC, Graybiel AM (2009) Stable encoding of task structure coexists with flexible coding of task events in sensorimotor striatum. J Neurophysiol 102:2142-2160.

Lau B, Glimcher PW (2007) Action and outcome encoding in the primate caudate nucleus. J Neurosci 27:14502-14514.

Luk KC, Sadikot AF (2001) GABA promotes survival but not proliferation of parvalbumin-immunoreactive interneurons in rodent neostriatum: an in vivo study with stereology. Neuroscience 104:93-103.

Mallet N, Le Moine C, Charpier S, Gonon F (2005) Feedforward inhibition of projection neurons by fast-spiking GABA interneurons in the rat striatum in vivo. J Neurosci 25:3857-3869.

McHaffie JG, Stanford TR, Stein BE, Coizet V, Redgrave P (2005) Subcortical loops through the basal ganglia. Trends Neurosci 28:401-407.

McKown MD, Schadt JC (2006) A modification of the Harper-McGinty microdrive for use in chronically prepared rabbits. J Neurosci Methods 153:239-242.

Munoz DP, Fecteau JH (2002) Vying for dominance: dynamic interactions control visual fixation and saccadic initiation in the superior colliculus. Prog Brain Res 140:3-19.

Nagy A, Eordegh G, Norita M, Benedek G (2003) Visual receptive field properties of neurons in the caudate nucleus. Eur J Neurosci 18:449-452.

Nagy A, Eordegh G, Paroczy Z, Markus Z, Benedek G (2006) Multisensory integration in the basal ganglia. Eur $\mathrm{J}$ Neurosci 24:917-924.

Nagy A, Paroczy Z, Markus Z, Berenyi A, Wypych M, Waleszczyk WJ, Benedek G (2008) Drifting grating stimulation reveals particular activation properties of visual neurons in the caudate nucleus. Eur J Neurosci 27:1801-1808.

Nagypal T, Gombkoto P, Barkoczi B, Benedek G, Nagy A (2015) Activity of caudate nucleus neurons in a visual fixation paradigm in behaving cats. PLoS One 10:e0142526.
Nagypal T, Gombkoto P, Utassy G, Averkin RG, Benedek G, Nagy A (2014) A new, behaving, head restrained, eye movementcontrolled feline model for chronic visual electrophysiological recordings. J Neurosci Methods 221:1-7.

National Research Council (U.S.) (2011) Committee for the Update of the Guide for the Care and Use of Laboratory Animals., Institute for Laboratory Animal Research (U.S.), National Academies Press (U.S.) (2011) Guide for the care and use of laboratory animals. Washington, D.C.: National Academies Press. p. 220.

Pelli DG (1997) The VideoToolbox software for visual psychophysics: transforming numbers into movies. Spat Vis 10:437-442.

Phelps PE, Houser CR, Vaughn JE (1985) Immunocytochemical localization of choline acetyltransferase within the rat neostriatum: a correlated light and electron microscopic study of cholinergic neurons and synapses. J Comp Neurol 238:286-307.

Pigarev IN, Levichkina EV (2011) Distance modulated neuronal activity in the cortical visual areas of cats. Exp Brain Res 214:105-111.

Pigarev IN, Rodionova El (1998) Two visual areas located in the middle suprasylvian gyrus (cytoarchitectonic field 7) of the cat's cortex. Neuroscience 85:717-732.

Populin LC, Yin TC (1998) Behavioral studies of sound localization in the cat. J Neurosci 18:2147-2160.

Populin LC, Yin TC (2002) Bimodal interactions in the superior colliculus of the behaving cat. J Neurosci 22:2826-2834.

Pouderoux G, Freton E (1979) Patterns of unit responses to visual stimuli in the cat caudate nucleus under chloralose anesthesia. Neurosci Lett 11:53-58.

Rajkai C, Lakatos P, Chen CM, Pincze Z, Karmos G, Schroeder CE (2008) Transient cortical excitation at the onset of visual fixation. Cereb Cortex 18:200-209.

Robinson DA (1963) A method of measuring eye movement using a scleral search coil in a magnetic field. IEEE Trans Biomed Eng 10:137-145.

Rokszin A, Gombkoto P, Berenyi A, Markus Z, Braunitzer G, Benedek G, Nagy A (2011) Visual stimulation synchronizes or desynchronizes the activity of neuron pairs between the caudate nucleus and the posterior thalamus. Brain Res 1418:52-63.

Rolls ET, Thorpe SJ, Maddison SP (1983) Responses of striatal neurons in the behaving monkey. 1. Head of the caudate nucleus. Behav Brain Res 7:179-210.

Schmitzer-Torbert N, Redish AD (2004) Neuronal activity in the rodent dorsal striatum in sequential navigation: separation of spatial and reward responses on the multiple $T$ task. J Neurophysiol 91:2259-2272.

Schmitzer-Torbert NC, Redish AD (2008) Task-dependent encoding of space and events by striatal neurons is dependent on neural subtype. Neuroscience 153:349-360.

Stalnaker TA, Calhoon GG, Ogawa M, Roesch MR, Schoenbaum G (2012) Reward prediction error signaling in posterior dorsomedial striatum is action specific. J Neurosci 32:10296-10305.

Strecker RE, Steinfels GF, Abercrombie ED, Jacobs BL (1985) Caudate unit activity in freely moving cats: effects of phasic auditory and visual stimuli. Brain Res 329:350-353.

Sumner P, Nachev P, Castor-Perry S, Isenman H, Kennard C (2006) Which visual pathways cause fixation-related inhibition? J Neurophysiol 95:1527-1536.

Tepper JM, Tecuapetla F, Koos T, Ibanez-Sandoval O (2010) Heterogeneity and diversity of striatal GABAergic interneurons. Front Neuroanat 4:150.

Thorn CA, Atallah H, Howe M, Graybiel AM (2010) Differential dynamics of activity changes in dorsolateral and dorsomedial striatal loops during learning. Neuron 66:781-795.

Tollin DJ, Populin LC, Moore JM, Ruhland JL, Yin TC (2005) Soundlocalization performance in the cat: the effect of restraining the head. J Neurophysiol 93:1223-1234.

Velly LJ, Rey MF, Bruder NJ, Gouvitsos FA, Witjas T, Regis JM, Peragut JC, Gouin FM (2007) Differential dynamic of action on cortical and subcortical structures of anesthetic agents during induction of anesthesia. Anesthesiology 107:202-212. 
Vicente AF, Bermudez MA, Romero Mdel C, Perez R, Gonzalez F (2012) Putamen neurons process both sensory and motor information during a complex task. Brain Res 1466:70-81.

Villeneuve MY, Casanova C (2003) On the use of isoflurane versus halothane in the study of visual response properties of single cells in the primary visual cortex. J Neurosci Methods 129:19-31.
Wilson CJ (1993) The generation of natural firing patterns in neostriatal neurons. Prog Brain Res 99:277-297.

Wurtz RH (1969) Visual receptive fields of striate cortex neurons in awake monkeys. J Neurophysiol 32:727-742.

(Received 21 December 2016, Accepted 15 May 2017)

(Available online 22 May 2017) 